TS-DET 93-035

June 9, 1993

\title{
High Voltage Ramp Study of Philips XP2012 and XP2008 PMT's
}

\begin{abstract}
S. Delchamps
Results are presented for a high voltage ramp study of three (3) XP2012 PMT's and one (1) XP-2008 PMT. These 1.5" diameter, 10-stage PMT's were used in the UA2 experiment and are candidates for some parts of the CDF plug upgrade calorimeter.
\end{abstract}

Three (3) Philips XP2012 PMT's and one (1) Philips XP2008 PMT, were tested for sensitivity of response to ramping of the high voltage from high voltages corresponding to absolute gain $2.5 \times 10^{\wedge} 5$ to $500 \mathrm{~V}$ and $0 \mathrm{~V}$, and then back again. The response of each PMT was measured in terms of its "normalized corrected response" or NCR [see note 1 for an explanation of this quantity], to flashes of light emerging from a $1 \mathrm{~mm}$ Bicron BCF91A wavelength shifter fiber about $10 \mathrm{~cm}$ long and a clear fiber about $1 \mathrm{~m}$ long. The flashes were produced with the laser-based PMT test system at Lab 2 [2]. The raw data files for these runs are stored in the central vax cluster area

FNALV::PRJ\$ROOT:[TS_CDF_PRJ.STABILITY.HV060193]

[See Note 3 for instructions on reproducing the plots and numerical data found in this memo.]

Test Cycles: This test was a continuation of a long stability run reported on in TS-DET 93-034. The zero of time for the plots in this memo is the start of the test in the earlier memo. Three ramp cycle tests were performed. Throughout the entire cycle, the high voltage of the monitor PMT (see below) was held fixed. At 0 hours, the PMT's were turned on to high voltage values corresponding to gains of $2.5 \times 10^{\wedge} 5$.

Ramp 1: At 96.87 hours, the high voltages of all PMT's were ramped down to $0 \mathrm{~V}$. At 98.38 hours, the voltages were ramped back up to the original values.

Ramp 2: At 105.55 hours, the voltages were ramped down to $500 \mathrm{~V}$. At 106.68 hours, they were ramped up to the original values.

Ramp 3: At 113.83 hours the high voltages were ramped down to $500 \mathrm{~V}$, and at 114.96 hours they were ramped up again.

In all cases, the ramp-down and ramp-up times for the high voltages were on the order of seconds. After ramping the high voltages back up to their nominal values, data were taken at first every 3-4 minutes. After approximately 2.5 hours, data were taken every 10-12 minutes until the next ramp-down.

Monitor PMT: A single Hamamatsu R580-17 PMT was used to monitor the laser light intensity on a flash to flash basis. The monitor PMT (serial number WA2216) was placed on a separate high voltage supply, so that when the other PMT high voltages were ramped, the voltage of the monitor tube would not be affected. Figure la shows the response of the monitor PMT, normalized to its initial value, as a function of time during the test. Variations in the response of the correction PMT reflect both actual changes in 
laser intensity and any intrinsic variations in the gain times quantum efficiency of the monitor PMT.

Figure $1 \mathrm{~b}$ shows the NCR for a PIN diode. This diode was connected to a separate power supply, so that its NCR was expected to be stable throughout the test. It is therefore interesting and rather disturbing that the PIN diode NCR showed large scatter, and two large dips near the times at which the temperature in the test chamber as measured by probe $\mathrm{B}$ had dips.

Figures $1 \mathrm{c}$ and $1 \mathrm{~d}$ show the temperature inside the test chamber, as recorded by two independent thermometers. One of the temperature probes, $\mathrm{Tb}$, shows pronounced dips during the intervals during which the high voltages were turned off. Tb was placed close to the PMT bases in the test chamber.

Ramp Test Results: Figures 2a - 2d show the NCR's for the Philips PMT's under test. The earliest NCR values in the plots appear to lie systematically high relative to the NCR values after the first ramp-down, at about 96 hours. (Data were not taken between the first ramp-down and the first ramp-up.)

For times between 90 and 96 hours, the NCR values come from the stability run reported on in TS-DET 93-034, in which an unexplained upturn in the NCR values toward the end of the run was apparent. In the present test, it is seen that the NCR's after the first ramp-up (at about 98 hours) do not approach the pre-ramp-down value, but rather a value somewhat lower, which may correspond to the "true" NCR value before the ramp-down. However, the data here were analyzed as if the NCR values shown in the figures were correct.

The XP2012's with serial numbers 12001 and 12002 have NCR's which start a few percent higher than their pre-ramp-down value. The XP2012 with serial number 12003 and the XP2008 PMT have NCR's which start closer to their pre-ramp-down values.

Results are summarized in Table 1. The first two columns give the PMT types and serial numbers, including the PIN diode. The last three columns correspond to the three ramp tests. Three numbers are given for each PMT: 1) The percent of the pre-ramp-down NCR measured just after the high voltage is restored, 2) The time in minutes required for the NCR to return to within $1 \%$ of its pre-ramp-down value, and 3 ) The time in minutes required for the NCR to return to within $2 \%$ of its pre-ramp-down value. The minimum time possible is 3.0 minutes; this is because of the non-zero deadtime for taking data. Thus, a PMT reporting 3.0 minutes for the $1 \%$ return time is, to within our resolution, returning to its pre-ramp-down NCR value instantaneously when returned to its nominal voltage.

PMT's 12001 and 12002 in general took much longer than the other PMT's to return to their pre-ramp-down values. 


\begin{tabular}{|c|c|c|c|c|c|c|c|c|c|c|}
\hline \multirow{2}{*}{$\begin{array}{l}\text { PMT } \\
\text { Type }\end{array}$} & \multirow[t]{2}{*}{ Serial Number } & \multicolumn{3}{|c|}{ Test Cycle 1} & \multicolumn{3}{|c|}{ Test Cycle 2} & \multicolumn{3}{|c|}{ Test Cycle 3} \\
\hline & & $\begin{array}{l}\% \text { of } \\
\text { pre- } \\
\text { ramp }\end{array}$ & $\begin{array}{l}\Delta \mathrm{t} \text { for } \\
1 \% \\
\text { (min.) }\end{array}$ & $\begin{array}{c}\Delta \mathrm{t} \text { for } \\
2 \% \\
\text { (min.) }\end{array}$ & $\begin{array}{l}\% \text { of } \\
\text { pre- } \\
\text { ramp }\end{array}$ & $\begin{array}{l}\Delta \mathrm{t} \text { for } \\
1 \% \\
\text { (min.) }\end{array}$ & $\begin{array}{c}\Delta \mathrm{t} \text { for } \\
2 \% \\
\text { (min.) }\end{array}$ & $\begin{array}{l}\% \text { of } \\
\text { pre- } \\
\text { ramp }\end{array}$ & $\begin{array}{l}\Delta \mathrm{t} \text { for } \\
1 \% \\
\text { (min.) }\end{array}$ & $\begin{array}{l}\Delta \mathrm{t} \text { for } \\
2 \% \\
\text { (min.) }\end{array}$ \\
\hline XP-2012 & 12001 & 104.7 & 23.4 & 13.2 & 101.5 & 6.0 & 3.0 & 102.8 & 17.4 & 7.2 \\
\hline & 12002 & 111.6 & 42.0 & 36.0 & 106.3 & 36.0 & 22.0 & 106.9 & 46.2 & 20.4 \\
\hline & 12003 & 99.9 & 3.0 & 3.0 & 99.9 & 3.0 & 3.0 & 100.4 & 3.6 & 3.6 \\
\hline XP-2008 & 8002 & 99.7 & 3.0 & 3.0 & 99.6 & 3.0 & 3.0 & 100.0 & 3.6 & 3.6 \\
\hline
\end{tabular}

Table 1. Results from High Voltage Ramp Study of Philips PMT's 


\section{Notes}

1. The NCR or "normalized corrected response" is the "corrected response" (CR) divided by its initial value. The CR can be defined for a PMT as

$$
\begin{aligned}
& 500 \\
& \mathrm{CR}=\left\{\Sigma\left(\mathrm{PMT}_{\mathrm{i}} / \mathrm{CORR}_{\mathrm{i}}\right)\right\} / 500 \\
& \mathrm{i}=1
\end{aligned}
$$

where $\mathrm{PMT}_{\mathrm{i}}$ is the pedestal-subtracted response of the PMT for the ith flash, and CORR is the response of the correction device (in the case of the test reported in this memo, the monitor PMT WA2216), for the ith flash. The correction device in principle removes the variations in the PMT response which are due only to variations in the laser light level. It is assumed that the correction device is perfectly stable, and that the ratio

$$
\mathrm{R}=\text { Light delivered to the correction device/ Light delivered to the PMT }
$$

for each laser flash is a constant. (See TS-DET 93-002 for more details.)

2. S. Delchamps, and B. Kinney. "Preliminary PMT Stability Measurements with Laser Light Source." TS-DET 93-002.

3. To reproduce the plots in this memo, go to the area on the central VAX cluster shown on the first page. Once there, execute the command procedure LASER.COM. Next, execute the procedure HVTEST.COM. This will produce three ascii files, TABLE1-3.DAT, which contain the information from Table 1. To produce the plots, execute the file LASER.KUMAC from inside of PAW.

4. See TS-DET 93-034 for stability measurements of these PMT's. 


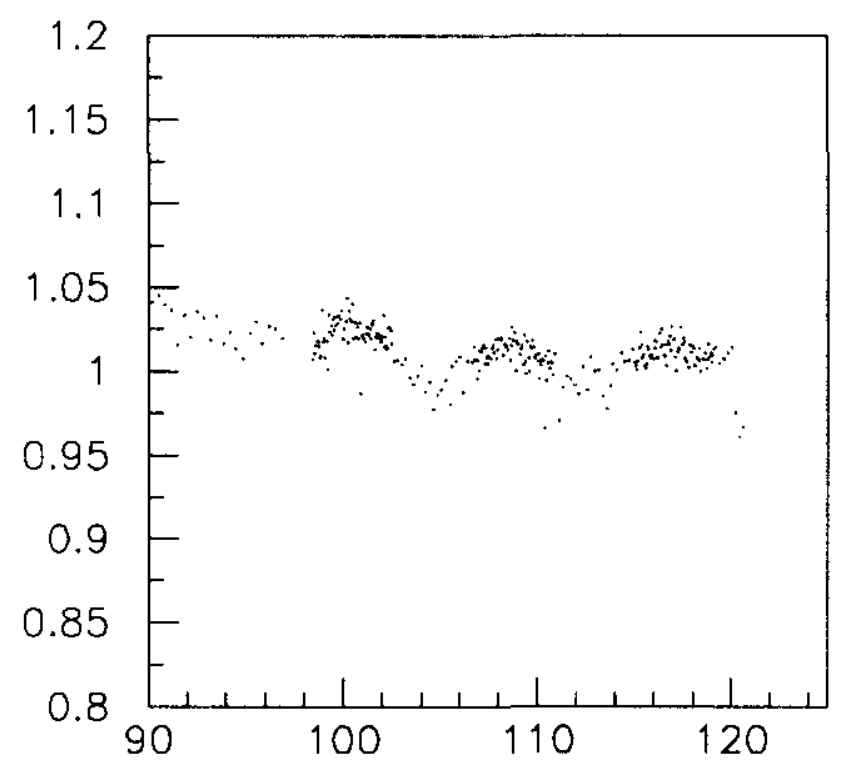

Fig. 1 a WA2216 vs Time (hrs)

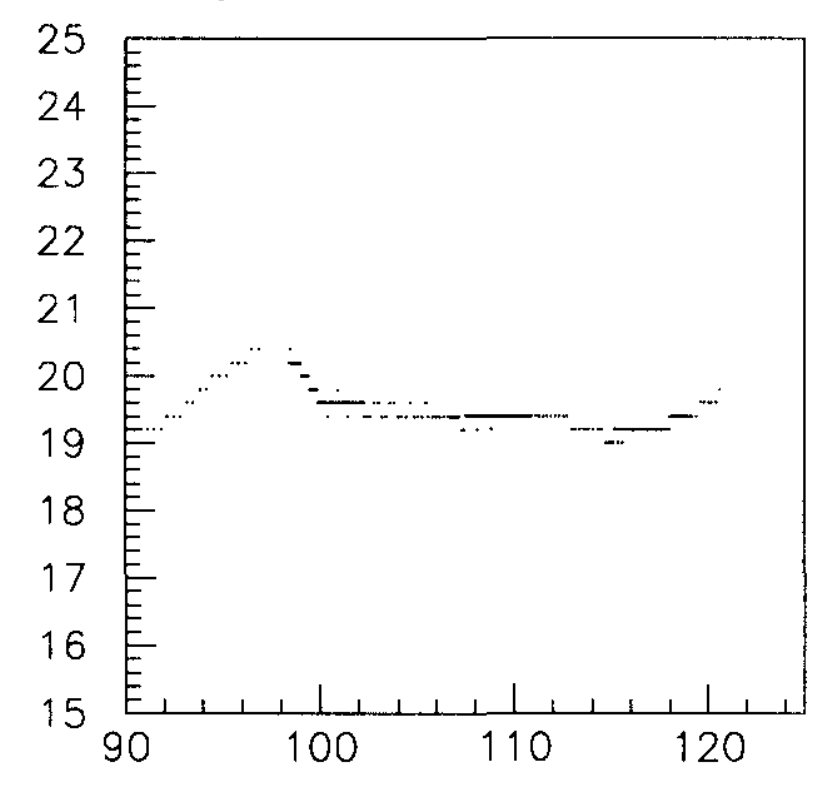

Fig. 1c Ta vs Time (hrs)

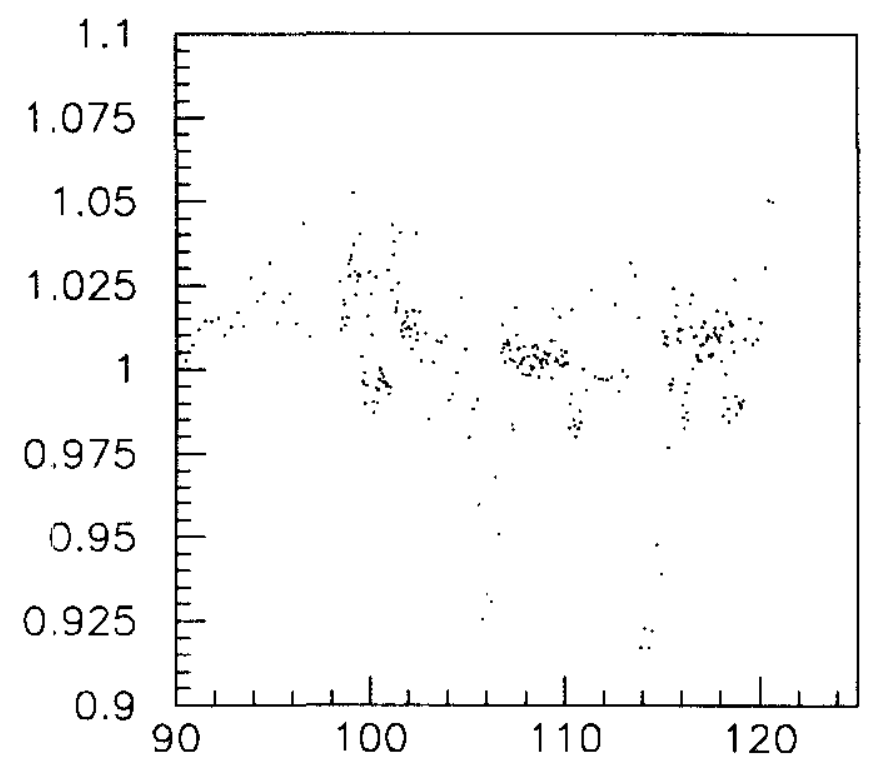

Fig. 1b PIN 2 vs Time (hrs)

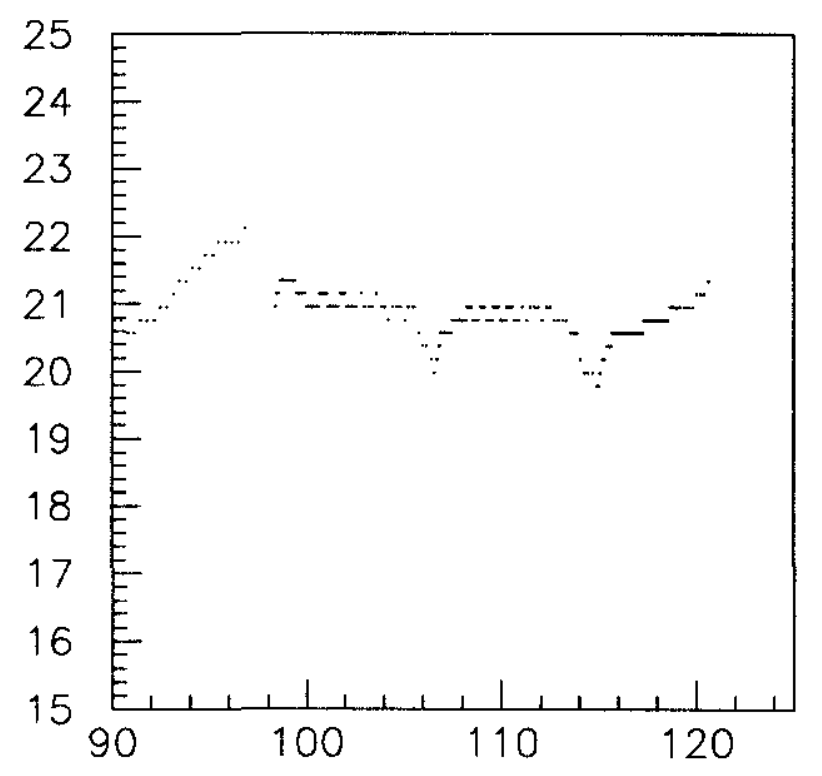

Fig. 1d Tb vs Time (hrs) 




Fig. 2o 12001 vs Time (hrs)

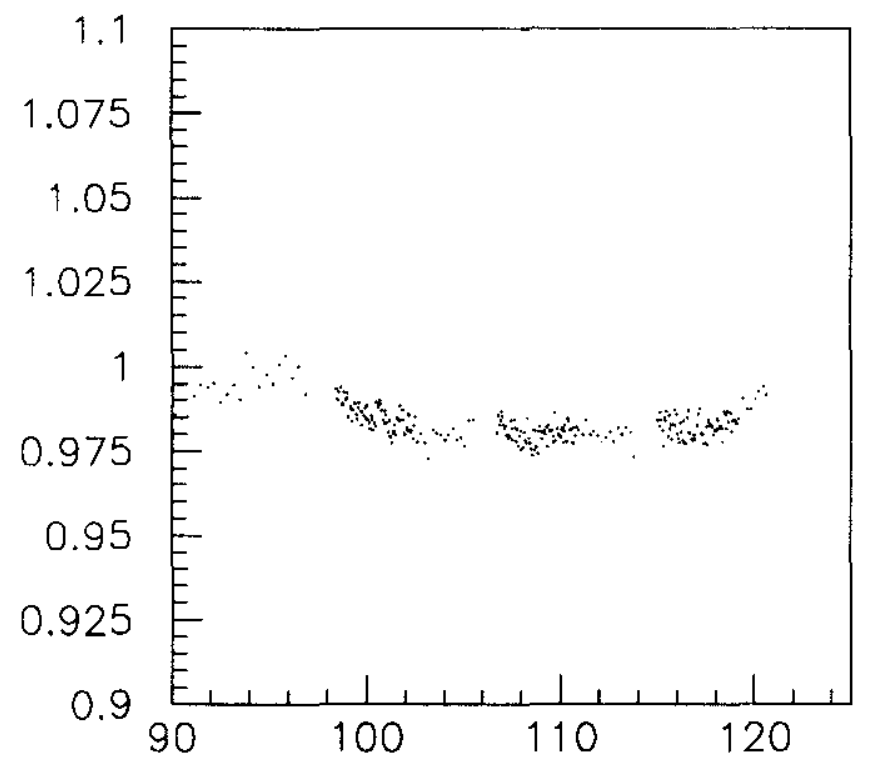

Fig. 2c 12003 vs Time (hrs)

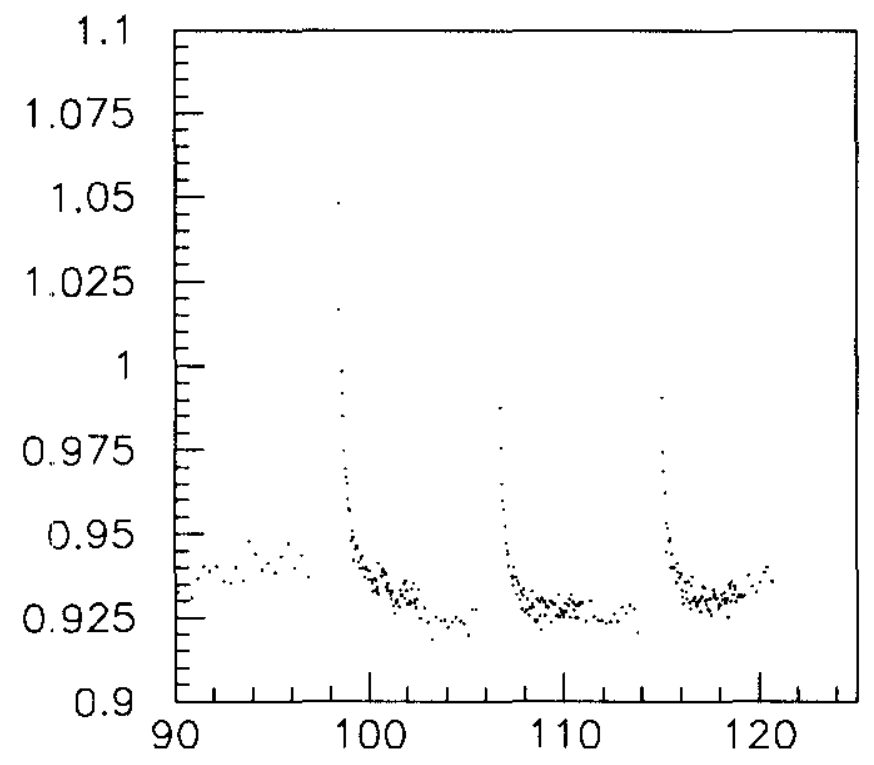

Fig. 2b 12002 vs Time (hrs)

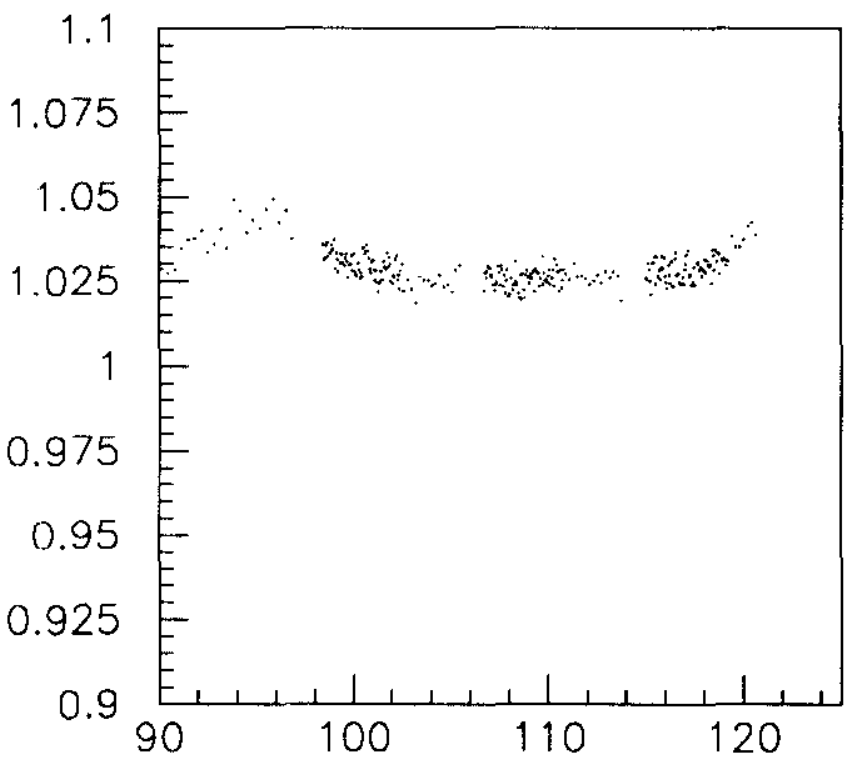

Fig. 2d 8002 vs Time (hrs) 